\title{
A QUALIDADE DE VIDA DE PESSOAS COM DIABETES MELLITUS DE UM MUNICÍPIO NO INTERIOR DO RIO GRANDE DO SUL
}

\author{
QUALITY OF PEOPLE LIVING WITH DIABETES MELLITUS \\ IN A CITY IN THE INTERIOR OF RIO GRANDE DO SUL
}

\section{Tayane Silva da Silva', Aline Sarturi Ponte², Jodeli Pommerehn ${ }^{3}$, Miriam Cabrera Corvelo Delboni ${ }^{4}$}

\section{RESUMO}

A Diabetes Mellitus (DM) é uma doença crônica não transmissível que exige tratamento, controle, mudanças de hábitos e rotina, estes fatores causam impactos no cotidiano e na Qualidade de Vida (QV) dos sujeitos acometidos pela doença. Objetivo: Avaliar a QV de pessoas com DM usuárias de um serviço ambulatorial hospitalar localizado na região central do Estado do Rio Grande do Sul, RS/Brasil. Metodologia: Pesquisa quantitativa do tipo descritiva baseada na correlação de Pearson. Utilizaram-se dois instrumentos para coleta de dados, o Questionário de Informações Sociodemográficas e a Escala Paid. Na análise dos dados utilizou-se o software Statistical Package for Social Science 15.0 (SPSS). Resultados: A amostra foi composta por de 20 sujeitos, sendo que $60 \%$ de indivíduos do sexo masculino, com média de idade de $59,25( \pm 10,38)$ anos e $40 \%$ do sexo feminino, idade média de $60,00( \pm 8,12)$ anos. A dimensão definida como a mais problemática pelos entrevistados foi a que se refere a problemas emocionais relacionados ao diabetes. Conclusão: O tempo para o início do tratamento, apoio social, fatores emocionais e a preocupação com as consequências da doença (principalmente as amputações e ulceras) apresentaram-se neste estudo como fatores que influenciam na QV dos sujeitos após o diagnóstico de DM.

Descritores: Diabetes Mellitus; Qualidade de Vida; Percepção Social; Atenção Primária à Saúde.

\section{ABSTRACT}

Diabetes Mellitus (DM) is a non-communicable chronic disease that requires treatment, control, habits and routine changes, these factors cause impacts on daily life and quality of life (QOL) of individuals affected by the disease. Objectives: Evaluate the QOL of people with DM attending a hospital outpatient clinic located in the central region of the State of Rio Grande do Sul, RS/Brazil. Methodology: Quantitative descriptive research based on Pearson correlation. They use two instruments for data collection, the Sociodemographic Information Questionnaire and Scale Paid. In the data analysis used the software Statistical Package for Social Science 15.0 (SPSS). Results: The sample consisted of 20 subjects, $60 \%$ of males, with a mean age of $59.25( \pm 10.38)$ years and $40 \%$ female, mean age $60.00( \pm 8,12)$ years. The dimension defined as the most problematic for individuals was referred to emotional problems related to diabetes. Final Thoughts: The time for the start of treatment, social support, emotional factors and concern about the consequences of the disease (mainly amputations and ulcers) presented in this study as factors that influence the QoL of subjects after the diagnosis of DM.
1 Graduada em Terapia Ocupacional pela Universidade Federal de Santa Maria (UFSM), Santa Maria, RS, Brasil.

${ }^{2}$ Mestre em Distúrbios da Comunicação Humana pela Universidade Federal de Santa Maria (UFSM), Santa Maria, RS, Brasil.

${ }^{3}$ Mestranda em Distúrbios da Comunicação Humana na Universidade Federal de Santa Maria (UFSM), Santa Maria, RS, Brasil.

${ }^{4}$ Doutoranda em Desenvolvimento Regional na Universidade de Santa Cruz do Sul (UNISC), Santa Cruz do Sul, RS, Brasil. 


\section{Introdução}

Dados apresentados pela Organização Mundial da Saúde (OMS), estimam que existam mais de 347 milhões de pessoas diagnosticadas com Diabetes Mellitus (DM) ${ }^{1}$. Estudos realizados no ano de 2013, pela Federação Internacional do Diabetes (IDF) indicaram que 11,9 milhões de pessoas com idade entre 20 e 79 anos foram diagnosticadas com DM no Brasil ${ }^{2}$, este dado coloca o Brasil em quarto lugar no ranking da doença no mundo ${ }^{3}$. A DM é um doença de múltipla etiologia, classificada com uma Doenças Crônicas Não Transmissíveis (DCNT) ${ }^{4}$, divide-se em dois tipos a Diabetes Mellitus tipo 1 (DM1) e o Diabetes Mellitus tipo 2 (DM2) 5 .

$O$ aumento da longevidade da população brasileira provocou importantes mudanças no perfil epidemiológico da população, com predomínio das DCNT sobre as doenças infecciosas e parasitárias ${ }^{6,7}$. Levantamentos do Ministério da Saúde, apontam que a proporção de mortes por DCNT triplicou entre 1930 e 2006, e que os principais fatores de risco são as doenças do aparelho circulatório a Hipertensão Arterial Sistêmica (HAS) e o DM8.

A DM "vem aumentando, o que se caracteriza como uma epidemia mundial e um problema de saúde pública"::485, resultante da deficiência e/ou da incapacidade da insulina atuar adequadamente no organismo. A insulina é um hormônio que regula o metabolismo dos carboidratos, lipídios e proteínas, portanto a carência desta no organismo resulta em um quadro de hiperglicemia que acaba danificando gravemente muitos órgãos e sistemas (especialmente nervos e vasos sanguíneos) ${ }^{5}$.

Os hábitos de vida (tabagismo, sedentarismo, hábitos alimentares inadequados, o consumo excessivo de bebidas alcoólicas) a obesidade, HAS e presença de doenças vasculares periféricas associadas a fatores como faixa etária, cuidado com membros inferiores e superiores, carência cuidado preventivo/educativo pós-diagnóstico e pouca eficiência dos Serviços de Atenção Primária em Saúde (APS), corroboram com a formação de lesões ulcerativas consequentemente infecções e gangrenas evoluindo para amputações de extremidades acometendo principalmente nos membros inferiores, resultando em incapacidade, invalidez (aposentadoria precoce) e até mesmo óbito 5 .

Após o diagnóstico de DM preconiza-se o acompanhamento dos sujeitos pela APS, em alguns casos estes sujeitos não recebem o cuidado de saúde necessário. Esta falta de atenção em saúde aumenta o risco de complicações da doença e contribui para sobrecarga dos níveis de Atenção Secundário e Terciário ${ }^{10}$. Devido à complexidade do DM, ela foi incorporada progressivamente na atenção às DCNT com enfoque ao cuidado multiprofissional. Mas apesar dos benefícios desta forma de cuidado para o controle metabólico e a redução dos fatores de riscos associados às complicações do DM, sua implantação ainda representa um grande desafio na saúde ${ }^{11}$.

Diante disso, sabe-se da necessidade do fortalecimento das ações de integrativas, previstas do Sistema Único de Saúde - SUS ${ }^{12}$. Tais ações com vistas a prevenir as complicações em saúde que demandem de cuidados em nível de média e alta complexidade (diminuindo os custos destes setores) ${ }^{13}$. 0 acompanhamento pela APS de pessoas com DM, preconizado pelo SUS, visa o controle da doença, através de conscientização, medicação, acompanhamento e orientação. Tais ações visam a prevenção e a Qualidade de Vida (QV) dos sujeitos ${ }^{14}$.

A definição de QV defendida pela OMS foi criada pelo WHOQOL Group e é resultado de um consenso internacional, representando uma perspectiva transcultural, bem como multidimensional, este grupo define QV como, "a percepção do indivíduo de sua posição na vida no contexto da cultura e sistema de valores nos quais ele vive e em relação aos seus objetivos, expectativas, padrões e preocupações"15:28.

A QV abrange o sujeito em toda a sua subjetividade, considerando a complexa influência da saúde física e psicológica, nível de independência, relações sociais, crenças pessoais e das suas relações com características salientes do respectivo meio ao qual o mesmo está inserido ${ }^{15,16-18}$.

Além das DCNT tornarem-se um problema de saúde pública elas também ameaçam a QV, pois interferem no cotidiano e em todos os aspectos da saúde física, social e psíquica das pessoas ${ }^{4}$. Sendo assim, avaliar a percepção da QV das pessoas com DM pode ser uma forma de compreendermos o impacto das modificações no cotidiano da pessoa que se descobre diabética.

Este estudo tem como objetivo avaliar a QV de pessoas com DM usuárias de um serviço ambulatorial hospitalar localizado na região central do estado do Rio Grande do Sul, RS. 


\section{Metodologia}

Trata-se de um estudo quantitativo transversal ${ }^{19}$. Todos os participantes assinaram um Termo de Consentimento Livre e Esclarecido (TCLE), o estudo foi desenvolvido após a aprovação do Comitê de Ética em Pesquisa (CEP) da Universidade Federal de Santa Maria (UFSM), sob o número de parecer 083632/2013, conforme a Resolução n $466 / 12$ do Conselho Nacional de Saúde (CNS), referente à pesquisa com seres humanos.

A coleta de dados ocorreu no período de fevereiro a agosto de 2013. Esta respeitou os seguintes critérios de inclusão, pessoas de ambos os sexos; idade igual ou superior a 18 anos (público atendido pelo Ambulatório Vascular de um hospital escola de uma universidade pública do interior do Rio Grande do Sul); com diagnóstico de DM tipos 1 ou 2; e que estivessem em atendimento no referido serviço durante a coleta de dados deste estudo.

Participaram do estudo deste estudo 20 sujeitos, estes foram entrevistados na sala de espera do Ambulatório Vascular do hospital, todos os questionários foram aplicados por uma das pesquisadoras. Os instrumentos utilizados na coleta foram um Questionário de Informações Sociodemográficas, elaborado pelas pesquisadoras e a Escala Problems Areas in Diabetes (PAID).

A PAID é um instrumento validado no Brasil, que avalia a partir da ótica dos sujeitos o impacto do DM (tipo 1 e 2) e do tratamento em suas vidas ${ }^{20}$. Este instrumento contém 20 questões relacionadas a viver com DM e seu tratamento, estas abordam aspectos emocionais (culpa, raiva, depressão, preocupação e medo) enfrentados por estes sujeitos ${ }^{20-21}$.

APAID produz um escore total que varia de $0-100$, onde um escore mais baixo (perto de 0 ) indica menor sofrimento emocional e o mais alto (próximos de 100) indica um alto nível de sofrimento emocional. Utiliza uma escala Likert de cinco pontos, estes variam de 0 a 4, onde, 0 não é um problema, 1 é um pequeno problema, 2 é um problema moderado, 3 é quase um problema sério e 4 é um problema sério. O escore total de 0-100 total é alcançado pela soma das respostas de 0-4 dadas nos 20 itens do PAID e multiplicando esta soma por 1,2520-21.

No Brasil foi realizado um estudo com 146 pessoas com DM tipo 2. Os resultados da pesquisa demonstraram boa consistência interna (Alfa de Cronbach $=0,93$ ). A versão brasileira da escala apresenta condições psicométricas e validade satisfatórias. Pode ser considerada como uma ferramenta de avaliação do impacto do DM na QV destes sujeitos ${ }^{20-21}$.

Para a análise dos dados foi utilizado o software Statistical Package for Social Science 15.0 (SPSS), com nível de confiança de $5 \%$.

Um dos procedimentos metodológicos utilizados foi a correlação de Pearson com este analisou-se as dimensões do PAID com as variáveis: idade, tempo de diagnóstico e tempo de tratamento.

$\mathrm{Na}$ avaliação da comparação de sexo (masculino e feminino) em relação as dimensões do PAID, foi testada a normalidade de cada uma variáveis e a analisado o tipo de teste a ser utilizado. Como eram dois grupos independentes com distribuição não normal foi aplicado o teste Mann Whitney. Igual procedimento foi realizado para avaliar a comparação às dimensões do PAID em relação a ter ou não lesão.

\section{Resultados}

Foram entrevistadas 20 pessoas com DM, a partir dos dados coletados traçou-se um perfil da população assistida no Ambulatório Vascular desta instituição. Este serviço presta atendimento a toda à região central do Rio Grande do Sul, $\mathrm{RS}$, sendo um serviço de referência no interior do Estado.

Os participantes eram de ambos os sexos, sendo que 12 (60\%) eram do sexo masculino e $8(40 \%)$ eram do sexo feminino (Tabela 1). A idade dos participantes deste variou entre 42 e 76 anos, a média de idade dos homens foi de 49,3 anos e das mulheres de 50,7 anos. Os sujeitos que tem DM tipo 1, tem uma média de idade de 59,6 anos, e tempo de conhecimento da doença igual ao tempo de tratamento, que nesse caso é de 12,5 anos. Já os sujeitos com de DM tipo 2, tem uma idade média de 59 anos, o tempo de tratamento é menor que o tempo de conhecimento, sendo que o tempo de conhecimento é de 11,5 anos, e tempo médio de tratamento de 10,8 anos.

A maioria dos participantes residia no de Santa Maria, RS (14 [70\%]) e $6(30 \%)$ residiam em outros municípios vizinhos. Quanto a atividade de trabalho, 14 (70\%) participantes estavam aposentado no momento do estudo e $6(30 \%)$ eram profissionalmente ativos, as atividades de trabalho mencionada pelos participantes foram doméstica, do lar e autônomo. 
Tabela 1 - Distribuição das variáveis sociodemográficas entre os participantes da pesquisa n=20.

\begin{tabular}{|c|c|c|}
\hline Variáveis & Frequência (n) & Percentual (\%) \\
\hline \multicolumn{3}{|l|}{ Sexo } \\
\hline Feminino & 8 & $40 \%$ \\
\hline Masculino & 12 & $60 \%$ \\
\hline \multicolumn{3}{|c|}{ Região de procedência } \\
\hline Santa Maria, RS & 14 & $70 \%$ \\
\hline Outros munícipios & 6 & $30 \%$ \\
\hline \multicolumn{3}{|c|}{ Profissionalmente Ativo } \\
\hline Não & 14 & $70 \%$ \\
\hline Sim & 6 & $30 \%$ \\
\hline \multicolumn{3}{|c|}{ Tipo de Diabetes Mellitus (DM) } \\
\hline DM 1 & 8 & $40 \%$ \\
\hline $\mathrm{DM} 2$ & 12 & $60 \%$ \\
\hline \multicolumn{3}{|l|}{ Hereditário } \\
\hline Não & 1 & $5,3 \%$ \\
\hline Sim & 19 & $94,7 \%$ \\
\hline \multicolumn{3}{|l|}{ Lesão no pé } \\
\hline Não & 10 & $50 \%$ \\
\hline Sim & 10 & $50 \%$ \\
\hline \multicolumn{3}{|c|}{ Outra Doença Associada } \\
\hline Não & 11 & $55 \%$ \\
\hline Sim & 9 & $45 \%$ \\
\hline \multicolumn{3}{|l|}{ Vista ACS' } \\
\hline Não & 8 & $40 \%$ \\
\hline Sim & 12 & $60 \%$ \\
\hline \multicolumn{3}{|c|}{ Cuidado com a Alimentação } \\
\hline Não & 6 & $30 \%$ \\
\hline Sim & 14 & $70 \%$ \\
\hline \multicolumn{3}{|l|}{ Atividade Física } \\
\hline Não & 11 & $55 \%$ \\
\hline Sim & 9 & $45 \%$ \\
\hline
\end{tabular}

'ACS - Agente Comunitário em Saúde

Em relação ao tipo de DM, a maioria relatou ter DM do tipo 2, sendo $12(60 \%)$ dos participantes e $8(40 \%)$ tem diagnóstico de DM do tipo 1. Apenas um participante relatou que a doença não é hereditária. Quanto as lesões decorrerem da DM metade (10 [50\%]) dos participantes mencionaram ter lesões relacionadas a essa doença, em sua totalidade, nos pés (Tabela 1). Destes 10 participantes 8 (40\%) foram submetidos a processos cirúrgicos para amputação.

Quanto a outras doenças, percebeu-se que $45 \%$ dos participantes tem alguma outra doença associada a DM, a mais comum, foi a HAS, acometendo 8 (40\%) participantes, as outras doenças associada foram relacionadas a problemas renais. 
Quando os participantes são questionados sobre os cuidados para controlar a DM, $14(70 \%)$ relataram ter algum cuidado nutricional e $6(30 \%)$ relaram não cuidar da alimentação. Referente as atividades físicas apenas 9 (45\%) mencionaram alguma prática de esportes e 11 (55\%) não praticam nenhum tipo de atividade física.

Na Tabela 2 pode-se observar que a dimensão definida como a mais problemática pelos participantes foi a que se refere a problemas emocionais relacionados ao DM, os itens o que pior foram avaliados dentro desta dimensão foram "Ficar brabo/irritado quando pensa em viver com diabetes" com média de 2,1 $( \pm 1,8)$, "Preocupar-se com o futuro e com a possibilidade de sérias complicações", com média de $2,4( \pm 1,7)$ e "Lidar com as complicações do diabetes" com média de 2,3 ( $\pm 1,8)$, logo esses itens são considerados pelos entrevistados como um problema moderado (Tabela 2).

Tabela 2 - Dimensões da escala PAID n=20.

\begin{tabular}{|c|c|c|c|c|c|}
\hline Dimensões e os itens correspondentes & $\mathbf{A}$ & Média & $\begin{array}{l}\text { Desvio } \\
\text { padrão }\end{array}$ & Mínimo & Máximo \\
\hline $\begin{array}{c}\text { Problemas emocionais relacionados ao } \\
\text { Diabetes Mellitus }\end{array}$ & 0,7 & 1,4 & 1,6 & & \\
\hline $\begin{array}{c}\text { Sentir medo quando pensa em viver } \\
\text { com Diabetes }\end{array}$ & & 0,8 & 1,6 & 0 & 4 \\
\hline $\begin{array}{c}\text { Ficar deprimido quando pensa em ter } \\
\text { que viver com Diabetes }\end{array}$ & & 0,4 & 1 & 0 & 4 \\
\hline $\begin{array}{l}\text { Não saber se seu humor ou sentimentos } \\
\text { estão relacionados com o seu Diabetes }\end{array}$ & & 1 & 1,4 & 0 & 4 \\
\hline $\begin{array}{c}\text { Preocupar-se com episódios de glicose } \\
\text { baixa }\end{array}$ & & 1,7 & 1,8 & 0 & 4 \\
\hline $\begin{array}{c}\text { Ficar brabo /irritado quando pensa em } \\
\text { viver com Diabetes }\end{array}$ & & 2,1 & 1,8 & 0 & 4 \\
\hline $\begin{array}{c}\text { Sentir que o seu Diabetes é um peso } \\
\text { para você }\end{array}$ & & 0,9 & 1,4 & 0 & 4 \\
\hline $\begin{array}{l}\text { Preocupar-se com o futuro e com a } \\
\text { possibilidade de sérias complicações }\end{array}$ & & 2,4 & 1,7 & 0 & 4 \\
\hline $\begin{array}{l}\text { Sentir-se culpado(a) ou ansioso(a) quando } \\
\text { você deixa de cuidar do seu Diabetes }\end{array}$ & & 1,1 & 1,5 & 0 & 4 \\
\hline Não aceitar seu Diabetes & & 1,8 & 1,9 & 0 & 4 \\
\hline $\begin{array}{l}\text { Sentir que o Diabetes está tomando } \\
\text { muito de sua energia mental e física } \\
\text { diariamente }\end{array}$ & & 1,1 & 1,4 & 0 & 4 \\
\hline Lidar com as complicações do Diabetes & & 2,3 & 1,8 & 0 & 4 \\
\hline $\begin{array}{c}\text { Sentir-se esgotado com o esforço } \\
\text { constante que é necessário para cuidar do } \\
\text { seu Diabetes }\end{array}$ & & 1,4 & 1,8 & 0 & 4 \\
\hline Problemas relacionados ao tratamento & 0,7 & 0,8 & 1,5 & & \\
\hline $\begin{array}{l}\text { A falta de metas claras e concretas no } \\
\text { cuidado do seu Diabetes }\end{array}$ & & 1,1 & 1,7 & 0 & 4 \\
\hline $\begin{array}{l}\text { Sentir-se desencorajado com o seu } \\
\text { tratamento do Diabetes }\end{array}$ & & 0,9 & 1,5 & 0 & 4 \\
\hline $\begin{array}{l}\text { Sentir-se insatisfeito com o médico que } \\
\text { cuida o seu Diabetes }\end{array}$ & & 0,5 & 1,2 & 0 & 4 \\
\hline
\end{tabular}

continua... 


\begin{tabular}{c|c|c|c|c|c}
\hline Dimensões e os itens correspondentes & $\mathbf{A}$ & Média & $\begin{array}{c}\text { Desvio } \\
\text { padrão }\end{array}$ & Mínimo & Máximo \\
\hline Problemas relacionados a alimentação & $\mathbf{0 , 8}$ & $\mathbf{1 , 4}$ & $\mathbf{1 , 7}$ & & \\
\hline $\begin{array}{c}\text { Enfrentar situações sociais relacionadas } \\
\text { aos cuidados com o Diabetes (por } \\
\text { exemplo: pessoas falando para você o que } \\
\text { você deve comer) }\end{array}$ & & 1 & 1,6 & 0 & 4 \\
\hline $\begin{array}{c}\text { Ter sentimentos de privação a respeito } \\
\text { da comida e refeições }\end{array}$ & & 1,5 & 1,7 & 0 & 4 \\
\hline $\begin{array}{c}\text { Preocupar-se com a comida e o que } \\
\text { comer }\end{array}$ & & 1,6 & 1,87 & 0 & 4 \\
\hline Problemas relacionados ao apoio social & $\mathbf{0 , 7}$ & $\mathbf{0 , 5}$ & $\mathbf{1 , 2}$ & & \\
\hline Sentir-se sozinho com seu Diabetes & & 0,4 & 1,9 & 0 & 4 \\
\hline $\begin{array}{c}\text { Sentir que seus amigos e familiares não } \\
\text { apoiam seus esforços em lidar com o seu } \\
\text { Diabetes }\end{array}$ & & 0,6 & 1,3 & 0 & 4 \\
\hline TOTAL & $\mathbf{0 , 8}$ & & & & \\
\hline
\end{tabular}

Na Tabela 3 verifica-se que a média do escore geral é $31,3( \pm 22,1)$ pontos. Sendo o valor mínimo de 0,0 referente a um participante que não mencionou problemas nos itens do PAID, enquanto que outro participante referiu problemas sérios em quase todos os itens. Para este segundo participante, apenas o item relacionado a "Ficar deprimido quando pensa em ter que viver com Diabetes" foi avaliado como não sendo um problema e o item "Sentir-se insatisfeito com 0 médico que cuida o seu Diabetes" foi avaliado como um problema moderado, levando a um escore geral de 92,5 pontos.

Tabela 3 - Análise do escore geral n=20.

\begin{tabular}{c|c|c|c|c}
\hline & Média & Desvio Padrão & Mínimo & Máximo \\
\hline Tratamento & 3,1 & 4,3 & 0 & 15 \\
\hline Alimentação & 5,2 & 5,8 & 0 & 15 \\
\hline Social & 1,3 & 2,5 & 0 & 10 \\
\hline Emocional & 21,6 & 12,7 & 0 & 55 \\
\hline Escore Geral & $\mathbf{3 1 , 3}$ & $\mathbf{2 2 . 1}$ & $\mathbf{0}$ & $\mathbf{9 2 , 5}$ \\
\hline
\end{tabular}

Na Tabela 4 pode-se verificar a correlação do escore total da escala PAID com as variáveis idades, tempo de diagnóstico e tempo de tratamento, observa-se que não existe correlação significativa entre elas, isto é, nem a idade, nem o tempo de diagnóstico e nem o tempo de tratamento tem influência no escore geral. Observar que as dimensões tratamento, alimentação, social e emocional também não apresentam correlação com as variáveis citadas anteriormente.

Tabela 4 - Correlação do escore PAID com idade, tempo de diagnóstico e tempo de tratamento n=20.

\begin{tabular}{c|c|c|c}
\hline & Idade & Tempo de Diagnóstico & Tempo de Tratamento \\
\hline Tratamento & $-0,07$ & 0,05 & 0,08 \\
\hline Alimentação & $-0,15$ & $-0,39$ & 0,37 \\
\hline Social & $-0,28$ & $-0,24$ & 0,2 \\
\hline Emocional & $-0,23$ & $-0,36$ & 0,37 \\
\hline Escore Geral & $\mathbf{- 0 , 2 3}$ & $\mathbf{- 0 , 3 3}$ & $\mathbf{- 0 , 3 2}$ \\
\hline
\end{tabular}

*correlação de Pearson 
Na Tabela 5 verifica-se que não existe diferença significativa entre homens e mulheres em relação a três dimensões definidas pelo PAID, são estas: alimentação, tratamento, emocional. Apenas a dimensão relacionada a parte social teve diferença significativa, sendo que a maior média é encontrada no sexo feminino.

Tabela 5 - Comparação das médias das dimensões em relação ao sexo dos participantes n=20.

\begin{tabular}{c|c|c|c|c|c}
\hline & Tratamento & Alimentação & Social & Emocional & Escore Geral \\
\hline \multicolumn{6}{|c}{ Sexo } \\
\hline Feminino & $2( \pm 2,3)$ & $5,1( \pm 6,3)$ & $2( \pm 1,8)$ & $21,4( \pm 9,2)$ & $30,6( \pm 17)$ \\
\hline Masculino & $3,8( \pm 5,3)$ & $5,3( \pm 5,6)$ & $0,8( \pm 2,8)$ & $21,7( \pm 15)$ & $31,7( \pm 25,7)$ \\
\hline P-valor & 0,6 & 0,8 & 0,2 & 0,7 & 0,8 \\
\hline Lesão & \multicolumn{6}{|c}{} \\
\hline Não & $3,6( \pm 4,1)$ & $5,1( \pm 6)$ & $2,1( \pm 3,1)$ & $20,7( \pm 14,4)$ & $30,6( \pm 24,9)$ \\
\hline Sim & $2,6( \pm 4,8)$ & $5,3( \pm 5,8)$ & $0,5( \pm 1,5)$ & $22,5( \pm 11,6)$ & $30( \pm 20,3)$ \\
\hline P-valor & 0,4 & 0,8 & 0,8 & 0,4 & 0,9 \\
\hline
\end{tabular}

\section{Discussão}

No presente estudo, a maioria dos participantes são homens (60\%) (Tabela 1), sendo assim o resultado não se assemelha com aos achados na literatura revisada ${ }^{22-28}$. No entanto, estudos realizados por Schmidt et a ${ }^{29}$, (utilizando-se de diferentes medidas bioquímicas para diagnóstico) em seis capitais brasileiras, identificam maior prevalência de DM entre os homens (42,6\%).

As discordâncias apresentadas pelos estudos citados anteriormente pode ser atribuídas a alguns fatores, tais como: a pouca preocupação dos homens com a sua saúde ${ }^{30}$, conceitos culturalmente constituídos (modelo de masculinidade), 0 horário de funcionamento é um fator que dificulta o acesso da população masculina aos serviços de saúde, pois, coincide com a sua carga horaria de trabalho diária, o medo de descobrir que tem algum problema de saúde, a vergonha de expor o corpo, principalmente os órgãos genitais, a carência de ações de saúde voltadas a aproximação da população masculina aos serviços de saúde, a formação da equipe profissional, a qual em sua maioria é constituída por mulheres ${ }^{33}$. Estas podem ser algumas das barreiras enfrentadas pelos homens no cuidado com a própria saúde.

A média de idade apresentadas pelos homens foi de 49,3 anos e das mulheres de 50,7 anos, ou seja, os participantes deste estudo em sua maioria foram diagnosticados com DM já na fase produtiva de suas vidas, tal como averiguado em um estudo realizado na região urbana de Montes Carlos, MG, ocupado em analisar a QV de 648 pessoas de ambos os sexos e várias faixas etárias, diagnosticas com Doenças Crônicas ${ }^{27}$. Já outra pesquisa nacional não corrobora com os dados apresentados neste estudo, esta também ocupada em discutir a QV, realizada em São Paulo, SP, com 100 participantes, ressalta que a faixa etária predominantes dos participantes foi de 60 a 69 anos de idade ${ }^{26}$.

Ao acometer sujeitos em profissionalmente ativo o DM e suas complicações podem comprometer a atividade laboral destes, levando ao absenteísmo no trabalho e em alguns casos até a aposentadoria precoce ${ }^{26,32-36}$ Esta condição de saúde aumenta de 60 a $70 \%$ as chances destes trabalhadores desenvolverem incapacidades para 0 trabalho, reduzindo assim substancialmente as chances do trabalhador se manter no mercado de trabalho ${ }^{33}$.

Em um estudo realizado nos anos de 2013 e 2014 pela Sociedade Brasileira de Diabete, o DM de tipo 1 está presente em $5 \%$ a $10 \%$ dos casos, já do DM de tipo 2 apresenta-se em $90 \%$ a $95 \%$ dos casos diagnosticados ${ }^{37}$. Sabe-se que a hereditariedade é um dos principais fatores de riscos relacionados ao DM, principalmente ao DM tipo 238. Neste estudo $94,7 \%$ dos participantes tem algum familiar próximo diagnosticado com a doença, tal como um estudo realizado em Palmeiras das Missões, RS, onde 78,9\% dos participantes associaram o diagnóstico de DM a hereditariedade ${ }^{39}$.

Quanto a adesão ao tratamento, observa-se que o tempo de conhecimento da doença pelos participantes com DM tipo 2 é diferente do tempo de tratamento, isto é, estes demoram quase 1 ano para iniciar o tratamento após o diagnóstico. Estudos realizados por Gross ${ }^{20}$ apontam que sujeitos com pouco tempo de diagnóstico do DM, apresentam maior dificuldades para lidar com questões relacionadas ao tratamento. 
Tal comportamento pode justificar-se pelo fato da doença crônica não apresentar sintomatologia específica em sua fase inicial, o que acarreta em um diagnostico tardio, de modo geral estes sujeitos tem uma percepção tardia quanto ao estar doente ${ }^{40}$. Em seus estudos Linck et al., ${ }^{40}$ refere que $50 \%$ das pessoas com doenças crônicas não aderem ao tratamento, gerando impacto negativo tanto na vida dos sujeitos acometidos pela DM quanto para o Estado.

O início tardio do tratamento pode desencadear agravos a condição de saúde destes sujeitos. $O$ pé diabético pode ser uma destas, sendo este responsável por $70 \%$ das amputações de membros, principalmente de membros inferiores ${ }^{4}$. Estudos realizados por Salomé, Blanes e Ferreira ${ }^{32}$, Jaksa e Mahoney ${ }^{41}$, Almeida et al. ${ }^{26}$, apontam que as lesões nos pés comprometem a capacidade funcional, impossibilitando-os, muitas vezes, de realizar suas atividades cotidianas.

Além das complicações funcionais, estas lesões também geram mudanças no estilo de vida, comprometem a autoestima, influenciando na QV dos sujeitos ${ }^{26}$. Um estudo realizado por Meneses et al., ${ }^{42}$ o qual utilizou-se do instrumento SF-36 para avaliar a QV, identificou que os sujeitos com DM e lesões nos membros inferiores apresentavam um baixa QV, sendo os domínios da QV mais comprometidos aqueles relacionados a capacidade funcional e a aspectos físicos, sociais e emocionais.

Os sujeitos com DM apresentam maior chance de desenvolver outras comorbidades, sendo a HAS a mais comum $^{43,44}$. A HAS colabora para o desenvolvimento e progressão das complicações crônicas do DM, pois está associada à intolerância à glicose, resistência à insulina, obesidade, dislipidemia e doença arterial coronariana. Fatores que contribuem para a formação de úlceras neuroisquêmicas e amputações das extremidades inferiores ${ }^{45}$.

Quanto ao cuidado em saúde os ACS desenvolvem um trabalho muito importante junto os sujeitos com DM, pois é de responsabilidades deste profissionais a realização do cadastramento das pessoas com DM no programa especifico de atenção à saúde desta população, o HIPERDIA(Sistema de Cadastramento Acompanhamento de Hipertensos e Diabéticos). A partir das visitas domiciliares estes profissionais tem a oportunidade de realizar orientações referentes aos cuidados que se deve ter diante da nova condição de saúde e monitorar estes sujeitos objetivando o não agravamento dos quadros ${ }^{46}$.

Estudos realizados por Anderson et al., ${ }^{47}$ ressaltam que o cuidado nutricional é um fator importantíssimo para 0 controle do DM, segundo estes autores a mudança na alimentação induzem a diminuição dos níveis séricos de glicose e insulina no período pós-prandial.

Além das mudanças nutricionais a American Diabetes Association (ADA) e European Association for the Study of Diabetes $(E A S D)^{48}$ após diversos estudos e discussões chegam ao consenso que as mudanças nutricionais (orientadas por um nutricionista), associadas às alterações no estilo de vida (prática de exercícios físicos), são essenciais para 0 controle do DM. Portanto torna-se necessário conscientizar o sujeito sobre a importância da adesão ao tratamento e aos cuidados com a saúde, assim resultando controle da doença.

Os problemas emocionais relacionados ao DM foram definidos, pelos participantes deste estudo, como a principal problemática. Estudos sugerem que quanto maior o estresse emocional relacionado ao DM, identificados pelos escores do B-PAID e suas subdimensões, maior será a insatisfação do sujeito ${ }^{20}$.

Pode-seassociar este resultadoas transformações cotidianas as quais os sujeitos com DM deparam-se após o diagnóstico. Pois esta a doença vem acompanhada de alterações nos hábitos de vida, como restrições alimentares, a necessidade de práticas de uma atividade física regular ${ }^{47}$, a administração de medicamentos, a necessidade de consultas médicas e exames regulares, são fatores que em alguns casos podem gera estresse emocional e frustrações para estes sujeitos ${ }^{49}$.

Sendo assim, após o diagnóstico do DM, percebe-se que os sujeitos sofrem transformações em sua rotina que comprometem a sua QV. Contudo, apesar do DM ser uma doença crônica, não significa que ela irá incapacitar de imediato os sujeitos por ela acometidos. Mas esta é uma doença que exige uma reorganização do cotidiano, mudanças em hábitos de vida e uma conscientização dos sujeitos sobre as suas responsabilidades com a própria saúde, objetivando a prevenção de complicações e a minimização dos agravos. Ressaltando que manutenção da QV está relacionada ao apoio social e aos componentes psicológicos do sujeito, ou seja, como este enfrenta a doença, seus efeitos e consequências ${ }^{49}$.

\section{Considerações Finais}

Observou-se neste estudo a prevalência de homens diagnosticados com Diabetes Mellitus. 0 tipo 2 da doença foi 0 mais frequente entre os participantes deste estudo, a hereditariedade foi um agravante apontado pelos mesmos. As lesões nos pés em decorrência da Diabetes Mellitus apresentaram-se em $50 \%$ dos casos levando a incapacidades funcionais e em alguns casos ao afastamento das atividades profissional como é o caso de seis participantes deste estudo. 
A adesão ao tratamento foi um aspecto relevante apresentado pelos participantes deste estudo. Percebeu-se que alguns demoraram em média um ano ou mais para aderir ao tratamento. Quanto aos cuidados com a saúde percebe-se que os participantes procuram aderir à mudança de hábitos alimentares, já a prática de exercícios físicos ainda não é muito frequente entre os mesmos.

O contexto que envolve o diagnóstico do Diabetes Mellitus (carga de informações sobre a doença, suas complicações, os cuidados com a saúde, as mudanças no estilo de vida, por exemplo) foi apontado pelos participantes deste estudo como geradores de estresse emocional. As mudanças drásticas no estilo de vida repercutem nos aspectos psíquicos e sociais, refletindo diretamente na Qualidade de Vida destes.

Pode-se concluir que embora a amostra de pessoas com Diabetes Mellitus obtida para este estudo seja modesta (fator considerado limitante deste estudo), o estudo apresentou escores relevantes para os problemas relacionados ao tempo de tratamento, apoio social e emocional. Devido à importância deste assunto, sugere-se que sejam realizados outros estudos com essa temática.

\section{Referências}

1. Organização Mundial da Saúde. 10 datos sobre la diabetes; 2012 [citado em: 07 jun 2013]. Disponível em: http://www. who.int/features/factfiles/diabetes/es/index.html

2. Federação Internacional do Diabetes. Diabetes Atlas 2013; 2013 [citado em: 07 jun 2016]. Disponível em: http://dro. deakin.edu.au/view/DU:30060687.

3. Klafke A, Duncan BB, Rosa RS, Moura L, Malta DC, Schmidt MI. Mortalidade por complicações agudas do diabetes melito no Brasil, 2006-2010. Epidemiol. Serv. Saúde. 2014; 23(3):455-462.

4. Brasil, Plano de ações estratégicas para o enfrentamento das doenças crônicas não transmissíveis (DCNT) no Brasil 2011-2022. Ministério da Saúde. Secretaria de Vigilância em Saúde. Departamento de Análise de Situação de Saúde. Brasília: Ministério da Saúde, 2011.

5. Marinho NBP, Vasconcelos HCA, Alencar AMPG, Almeida PC, Damasceno MMC. Diabetes Mellitus: fatores associados entre usuários da estratégia saúde da família. Acta Paul Enferm. 2012; 25(4):595-600.

6. Schramm JMA, Oliveira AF, Leite IC, Valente JG, Gadelha AMJ, Portela MC, et al. Epidemiological transition and the study of burden of disease in Brazil. Ciência \& Saúde Coletiva. 2004; 9(4):897-908.

7. Moura EC, Pacheco-Santos LM, Peters LR, Serruya SJ, Guimarães R. Research on chronic noncommunicable diseases in Brazil: meeting the challenges of epidemiologic transition. Revista Panamericana de Salud Publica. 2012; 31(3):240-245. 8. Malta DC, Cezáreo AC, Moura L, Moraes Neto OL, Silva JB Junior. A construção da vigilância e prevenção das doenças crônicas não transmissíveis no contexto do Sistema Único de Saúde. Epidemiol Serv Saúde. 2006;15(3):47-65.

9. Lima ACS, Araújo MFM, Freitas RWJF, Zanetti ML, Almeida PC, Damasceno MMC. Fatores de risco para diabetes mellitus tipo 2 em universitários: associação com variáveis sociodemográficas. Rev. Latino-Am. Enfermagem. 2014; 22(3):484-90.

10. Gomes R, Leal AF, Knauth D, Silva GSN. Sentidos atribuídos à política voltada para a Saúde do Homem. Ciênc. saúde coletiva. 2013; 17(10):2589-2596.

11. Costa MB, Castro APA. Abordagem interdisciplinar no tratamento do diabetes mellitus tipo 2: da teoria à prática. Extramuros: Revista de Extensão da Univasf. 2014; 1(2):30-7.

12. Brasil. Lei 8.080 de 19 de setembro de 1990. citado em: 10 jun 2016]. Disponível em: http://conselho.saude.gov.br/ legislacao/lei8080_190990.htm.

13. Moura LRP, Percepção do Usuário da Estratégia Saúde da Família Sobre o Atendimento de Urgência e Emergência. (Mestrado Profissional em Saúde da Família) - Centro Universitário UNINOVAFAPI, Terezina, PI, 2013.

14. Brasil. Linhas de cuidado: hipertensão arterial e diabetes. Brasília: Organização Pan-Americana da Saúde, 2010.

15. WHOQOL Group. Development of the WHOQOL: Rationale and current status. International Journal of Mental Health. 1994; 23(3):24-56.

16. WHOQOL Group. Study protocol for the World Health Organization project to develop a Quality of Life assessment instrument (WHOQOL). Quality of Life Research. 1993; 2:153-59.

17. WHOQOL Group. The World Health Organization Quality of Life Assessment (WHOQOL): Position paper from the World Health Organization. Social Science e Medicine. 1995; 41(10):1403-09. 
18. WHOQOL Group. The World Health Organization Quality of Life Assessment (WHOQOL): Development and general psychometric properties. Social Science e Medicine, 1998; 46(12):1569-85.

19. Lima-Costa MF, Barreto SM. Tipos de estudos epidemiológicos: conceitos básicos e aplicações na área do envelhecimento. Epidemiol. Serv. Saúde. 2003; 12 ( 4 ):189-201.

20. Gross, C. C. Versão Brasileira da Escala Paid (Problem Areas in Diabetes): Avaliação do Impacto do Diabetes na Qualidade de Vida. 62 f. Dissertação (Mestrado em Psicologia do Desenvolvimento) - UFGRS, Porto Alegre. 2004.

21. Gross CC, Scain SF, Scheffel R, Gross JL, Hutz CS. Brazilian version of the problem áreas in diabetes scale (B-PAID): validation and identification of individuals at high risk for emotional distress. Diabetes Res Clin Pratice. 2007; 76:455-59.

22. Salomé GM, Blanes L, Ferreira LM. Assessment of depressive symptoms in people with diabetes mellitus and foot ulcers. Rev Col Bras Cir. 2011; 38(5):327-33.

23. Meneses LC, Blanes L, Veiga DF, Gomes HC, Ferreira LM. Health related quality of life and self-esteem in patients with diabetic foot ulcers: results of a cross-sectional comparative study. Ostomy Wound Manage. 2011; 57(3):36-43.

24. Bezerra SMMS, Santos ICRV, Sobral PHAF, Dum GL. Pé diabético: um estudo do custo do tratamento de pacientes internados em hospital público da cidade de Recife. Nursing. 2011;13(154):161-5.

25. Faria HTG, Rodrigues FFL, Zanetti ML, Araújo MFM, Damaceno MMC.Fatores associados à adesão ao tratamento de pacientes com diabetes mellitus. Acta paul. enferm. 2013, 26(3):231-7.

26. Almeida AS, Silveira MM, Espírito Santo PF, Pereira RC, Salomé GM. Avaliação da qualidade de vida em pacientes com diabetes mellitus e pé ulcerado. Rev Bras Cir Plást. 2013;28(1):142-6.

27. Oliveira-Campos M, Rodrigues-Neto JF, Silveira MF, Neves DMR, Vilhena JM, Oliveira JF, Magalhães JC, Drumond D. Impacto dos fatores de risco para doenças crônicas não transmissiveis na qualidade de vida. Ciência \& Saúde Coletiva. 2013; 18(3):873-882.

28. Iser BPM, Stopa SR, Chieiri PS, Szwarcwald CL, Malta DC, Monteiro HOC, Duncan BB, Schmidt MI. Prevalência de diabetes autorreferido no Brasil: resultados da Pesquisa Nacional de Saúde 2013. Epidemiol. Serv. Saúde. 2015; 24(2):305-14. 29. Schmidt MI, Hoffmann JF, Diniz MFS, Lotufo PA, Griep RH, Bensenor IM, et al. High prevalence of diabetes and intermediate hyperglycemia - The Brazilian Longitudinal Study of Adult Health (ELSA-Brasil). Diabetol Metab Syndr. 2014; 6(123):1-9.

30. Petermann XB, Machado IS, Pimentel BN, Miolo SB, Martins LR, Fodosse E. Epidemiologia e cuidado à Diabetes Mellitus praticado na Atenção Primária à Saúde: uma revisão narrativa. Saúde (Santa Maria). 2015; 41(1):49-56.

31. Gomes R, Nascimento EF, Araujo FC. Por que os homens buscam menos os serviços de saúde do que as mulheres? As explicações de homens com baixa escolaridade e homens com ensino superior. Cad. Saúde Pública; 2007; 23(3):565-74.

32. Salomé GM, Blanes L, Ferreira LM. Functional capability of patients with diabetes with foot ulceration. Acta Paulista de Enfermagem. 2009; 22(4):412-6.

33. Herquelot E, Guéguen A, Bonenfant S, Dray-Spira R. Impact of diabetes on work cessation: data from the gazel cohort study. Diabetes Care. 2011; 34(6):1344-1349.

34. Salomé GM, Ferreira, LM. Qualidade de vida em pacientes com úlcera venosa em terapia compressiva por bota de Unna. Rev. Bras. Cir. Plást. 2012; 27(3):466-71.

35. Salomé GM, Blanes L, Ferreira LM. Avaliação de sintomas depressivos em pessoas com úlcera venosa. Revista Brasileira Cirurgia Plástica. 2012; 27(1):124-9.

36. Lobato BC, Teixeira CRS, Zanetti GG, Zanetti ML, Oliveira MD. Evidências das implicações do diabetes mellitus no trabalho: uma revisão integrativa. Rev. Eletr. Enf. 2014 out/dez;16(4):822-32.

37. Sociedade Brasileira de Diabetes. Diretrizes da Sociedade Brasileira de Diabetes: 2013-2014/ Sociedade Brasileira de Diabetes. - São Paulo: AC Farmacêutica, 2014.

38. Reis AF, Velho G. Bases Genéticas do Diabetes Mellitus Tipo 2. Arq Bras Endocrinol Metab. 2002; 46(4):426-32.

39. Küster, D. K., Lunkes, Â. C. D., Hesler, L. Z., Ruzin, S. C., Rodrigues, M. D. G. S. $O$ grupo como estratégia para promoção da saúde do diabético. Ciência, Cuidado e Saúde. 2013; 7(1):1-4.

40. Linck CL, Bielemann VLM, Souza AS, Lange C. Paciente crônico frente ao adoecer e a aderência ao tratamento. Acta paul. enferm. 2008; 21(2): 318-20.

41. Jaksa PJ, Mahoney JL. Quality of life in patients with diabetic foot ulcers: validation of the Cardiff Wound Impact Schedule in a Canadian population. Int Wound J. 2010;7(6):502-7.

42. Meneses LC, Blanes L, Veiga DF, Gomes HC, Ferreira LM. Healthrelated quality of life and self-esteem in patients with diabetic foot ulcers: results of a cross-sectional comparative study. Ostomy Wound Manage. 2011; 57(3):36-43. 
43. Hypertension in Diabetes Study Group. HDS 1: Prevalence of hypertension in newly presenting type 2 diabetic patients and association with risc factors for cardiovascular and diabetic complications. Journal of Hypertension, 1993; 11(3):309-17. 44. Viana MR, Rodriguez TT. Complicações cardiovasculares e renais no diabetes mellitus. R. Ci. med. biol., Salvador, 2011; 10(3):290-96. 45. Gamba MA, Gotlieb SLD, Bergamaschi DP, Vianna LAC. Amputações de extremidades inferiores por diabetes mellitus: estudo caso-controle. Revista de Saúde Pública, 2004; 38(3):399-404.

46. Souza MF, Hardt J. A assistência aos pacientes com diabetes mellitus tipo 2 sob a ótica dos agentes comunitários de saúde. Interbio. 2011; 5(1): 17-26.

47. Anderson JW, Randles KM, Kendall CWC, Jenkins DJA. Carbohydrate and fiber recommendations for individuals with diabetes: a quantitative assessment and meta analysis of the evidence. J Am Coll Nutr. 2004; 23(1): 5-17.

48. American Diabetes Association (ADA) and The European Association for the Study of diabetes (EASD). Management of Hyperglycemia in Type 2 Diabetes: A Consensus Algorithm for the Initiation and Adjustment of Therapy. A consensus statement from the American Diabetes Association (ADA) and the European Association for the Study of Diabetes (EASD). Diabetes Care. 2006; 28(8):1963-72.

49. Miranda LP, Gomes, LMX, Prado, PF, Barbosa TLA, Teles, MAB. Qualidade de vida de idosos com diabetes mellitus cadastrados na estratégia saúde da família. R. Min. Educ. Fís. 2010; 5(1):125-35.Itapevi (SP); 2009.

\section{Tayane Silva da Silva}

Endereço para correspondência - Av. Roraima, $n^{\circ} 1000$,

Prédio 26, sala 1306, Campus UFSM, Camobi, CEP 97015-900, Santa Maria, RS, Brasil.

E-mail: tay_01ssf@hotmail.com.

Lattes: http://lattes.cnpq.br/6108560365634522

Aline Sarturi Ponte - alinesarturi@hotmail.com

Jodeli Pommerehn - jodelipo@gmail.com

Miriam Cabrera Corvelo Delboni - miriamdelboni@gmail.com

\section{Enviado em 11 de março de 2016. Aceito em 11 de agosto de 2016.}


